\title{
LA UNIVERSIDAD DE LÉRIDA DURANTE EL PONTIFICADO DE ANTONIO AGUSTÍN (1561-1576)*
}

\author{
RAFAEL RAMIS BARCELÓ \\ Universitat de les Illes Balears - IEHM
}

\section{RESUMEN}

Este estudio presenta, en primer lugar, una nómina inédita de profesores de la Universidad de Lérida durante el pontificado de Antonio Agustín y se muestra la vinculación del obispo con la Universidad y su promoción del humanismo. En segundo lugar, a través de documentación que no se había estudiado, se analiza el grave conflicto entre el obispo, el Estudio General y los Paheres que desembocó en la reforma del Estudio llevada a cabo en 1575 .

Palabras clave: Antonio Agustín, Lérida, Estudio General, Humanismo, Profesorado.

\section{ABSTRACT}

This study presents, first of all, an unpublished list of professors of the University of Lérida during the pontificate of Antonio Agustín and shows the connection of the bishop with the University and his promotion of humanism. Secondly, through documentation that had not been studied, it is analyzed the serious conflict between the

* Edificio G. M. de Jovellanos. Universitat de les Illes Balears - Instituto de Estudios Hispánicos en la Modernidad (IEHM). Cra. de Valldemossa, km. 7.5, 07122 Palma de Mallorca. e-mail: r.ramis@, uib.es. En el artículo utilizaremos las siguientes siglas: ACA (Archivo de la Corona de Aragón), ACL (Arxiu Capitular de Lleida), AML (Arxiu Municipal de Lleida) y DGC (Dietaris de la Generalitat de Catalunya). 
bishop, the Studium Generale and the Paheres that led into the reform of the Study carried out in 1575 .

Keywords: Antonio Agustín, Lérida, Studium Generale, Humanism, Professorship.

Antonio Agustín Albanell (1517-1586) fue uno de los mayores humanistas y juristas de su época ${ }^{1}$. En este trabajo vamos a estudiar su vinculación con la Universidad de Lérida una época central en su vida: la de su etapa como obispo de Lérida, en la cual tuvo un papel fundamental en la promoción del profesorado del Estudio General y en la reforma de los Estatutos de dicha institución.

Analizar el Estudio General de Lérida es una empresa difícil, pues, como es sabido, la mayoría de la documentación de la Universidad desapareció con el traslado de la sede a Cervera, y casi todos los datos que actualmente se poseen proceden de las labores de reconstrucción de diferentes autores, entre los que destacan Ramon Gayá Massot ${ }^{2}$, Josep Lladonosa ${ }^{3}$ (que se ocupó preferentemente de los primeros siglos) y de Francesc Esteve Perendreu ${ }^{4}$ (quien consagró sus esfuerzos sobre todo a la época moderna).

El paso de Antonio Agustín ${ }^{5}$ por la mitra ilerdense ha sido estudiado en un sentido más histórico-filológico e histórico-artístico ${ }^{6}$ que en su dimensión reli-

1 Véase Esther Balasch (coord.), Antoni Agustí, bisbe de Lleida i arquebisbe de Tarragona, 1517-1586: aportacions entorn el marc sòcio-cultural de Catalunya en la seva època (Lleida: Publicacions dels Amics de la Seu Vella, 1995).

2 Ramon Gayá Massot, "Provisión de cátedras en el Estudio General de Lérida", Analecta Sacra Tarraconensia, 30 (1958): 233-296.

3 Josep Lladonosa, L'Estudi General de Lleida del 1430 al 1524 (Barcelona, Institut d'Estudis Catalans, 1970) así como también otras obras de carácter general: Lérida medieval. Tomo II (siglos XIV-XV), (Lérida: Dilagro, 1975); Lérida moderna. Epoca de los Austrias (Lérida: Dilagro, 1977); Lérida moderna. Epoca de los Borbones (Lleida: Dilagro, 1980) y muy especialmente, Història de Lleida, II (Lleida: Dilagro, 1991), 303-324.

4 Citemos sólo las más importantes: Francesc Esteve Perendreu, El Régimen jurídico del Estudio General de Lleida: s. XIII/XVIII (Lleida: Publicacions de l'Estudi General de Lleida-Pagès, 1992); Mestrescoles i rectors de l'Estudi General de Lleida (1597-1717) (Lleida: Edicions de la Universitat de Lleida, 2007) y El Col-legi universitari de l'Assumpció de Santa Maria de Lleida (segles XIV-XIX) (Lleida: Universitat de Lleida, 2010).

5 Para una biografía de Antonio Agustín, véase Joan Carbonell i Manils, Epigrafia i numismàtica a l'epistolari d'Antonio Agustín (1551-1563), Tesis doctoral, (Bellaterra: Universitat Autònoma de Barcelona, 1992), especialmente, 1-72.

6 Juan Francisco Alcina Rovira, "El Humanismo de Antonio Agustín", en A. Egido y J. E. Laplana (coord.), Mecenazgo y Humanidades en tiempos de Lastanosa: Homenaje a Domingo Ynduráin (Za-ragoza: Institución Fernando el Católico e Instituto de Estudios Altoaragoneses, 2008), 31-50. 
giosa, educativa e institucional. Es cierto que Lladonosa ${ }^{7}$ había indicado con acierto que la presencia de Antonio Agustín había favorecido el humanismo en el Estudio de Lérida, aunque no había precisado la nómina de los profesores ni la vinculación que tuvo con ellos.

Así como hay muy poca documentación del siglo XVI, el feliz hallazgo de una correspondencia y de documentación económica en los archivos ilerdenses nos permite una reconstrucción mucho más ajustada de esta etapa del Estudio y ponerla en relación con el episcopado de Antonio Agustín. En este estudio no sólo tenemos en cuenta datos aún no exhumados de los archivos de Lérida, sino también alguna información no analizada aún, procedente del Archivo de la Corona de Aragón, que resulta elocuente de la atención que prestaba Felipe II al Estudio ilerdense.

\section{LA ESTRUCTURA DE LA UNIVERSIDAD HASTA LA LLEGADA DE ANTONIO AGUSTÍN}

La Universidad de Lérida, creada en 1300 por Jaime II, ${ }^{8}$ mantuvo el modelo jurídico original durante las cuatro centurias en las que tuvo actividad: esencialmente, el autogobierno de los escolares (mediante la elección del Rector y otras atribuciones); el control municipal matizado por la existencia de un gobierno académico independiente que integraba las "naciones" de la Corona; un peso eclesiástico fuerte, que podía condicionar -aunque no obligar-a las autoridades universitarias; y, por último, una vocación monopolística frente a las demás Universidades creadas con posterioridad 9 .

La Universidad de Lérida, que había sido protegida y privilegiada por los diferentes monarcas, tuvo una fuerte competencia tanto de las Universidades fundadas en el siglo XV como de las sedes nuevas que habían emergido desde 1550, especialmente Barcelona y Zaragoza. El hecho de que Barcelona fuera Cap $i$ casal y que la administración del Principado hubiera crecido y estuviese fuertemente centralizada en la Ciudad Condal, favoreció las aspiraciones del

7 Josep Lladonosa, "Humanisme i Reformes a l'Estudi General de Lleida durant el segle XVI" en AAVV, VIII Congreso de Historia de la Corona de Aragón, III, 2, (Valencia: Universidad de Valencia, 1973), 87-106.

8 Mariano Peset, "La fundación y el fuero universitario de Lérida", Hispania, 199 (1998): 515536 y Juan Pemán Gavín, "Los estatutos fundacionales de la antigua universidad Ilerdense (año 1300)", en J. J. Busqueta (ed.), Llibre de les Constitucions i Estatuts de l'Estudi General de Lleida (Lleida: Universitat de Lleida, 2000), 25-53.

9 Esteve Perendreu, El régimen jurídico, 117-122. 
Estudio General barcelonés ${ }^{10}$, que fue adquiriendo cada vez más estudiantes de Artes y Medicina, y poco a poco fueron consolidando los estudios jurídicos y teológicos ${ }^{11}$.

La sede ilerdense tenía que luchar contra la competencia, pues a mediados del siglo XVI ya no tenía la situación privilegiada de la que había gozado en las centurias anteriores. La Universidad de Huesca asimismo le privó de alumnos del Reino de Aragón e incluso atrajo a muchos catalanes. Así como Carlos I había favorecido mucho la Ciudad Condal y su interés había resultado decisivo para desencallar las tensiones internas para su puesta en funcionamiento, su hijo Felipe II apoyó claramente la Universidad de Lérida ${ }^{12}$ como sede tradicional, especialmente como medio de protección frente las herejías propagadas en el sur de Francia ${ }^{13}$.

Tenemos escasos datos de los profesores ilerdenses en la época de Carlos I, si bien hemos podido espigar una nómina de 1535: Ferran de Cardona, Pere Soler, Pere Oriol, Pau Jordà, Miquel Miralles, Pere Robio, Onofre Vigelles, doctores en derecho, Joan Ozoro, Pere Joan Vilaplana, Gabriel Babot, Simó Pla, Joan Oroscaro, doctores en artes y medicina ${ }^{14}$. En 1536, como es sabido, se dio un gran impulso a la Facultad de Teología con la creación de la llamada cátedra de Conchillos ${ }^{15}$.

En Lérida, muchos de los profesores de leyes compatibilizaban sus cátedras con algunos cargos municipales, tales como la defensa jurídica de la ciudad, algo que, por ejemplo, estaba vetado en Barcelona. Sin embargo, el desempeño de cargos representativos en la Pahería era, en la práctica, del todo incompatible con la lectura de las cátedras, de manera que tenían que llamar a sustitutos para que se ocupasen de las mismas. También había muchos profesores eclesiásticos, que poseían al mismo tiempo una canongía u otro beneficio, o bien pertenecían al clero regular.

10 Antonio Fernández Luzón, La Universidad de Barcelona en el siglo XVI (Barcelona: Universitat de Barcelona, 2005).

11 Rafael Ramis Barceló, "Los graduados en Leyes y Cánones de la Universidad de Barcelona durante el siglo XVI", Anuario de Historia del Derecho Español 85 (2015): 475-496 y Rafael Ramis Barceló, "Los grados en teología en la Universidad de Barcelona durante el siglo XVI", Anuario de Historia de la Iglesia 24 (2015): 291-309.

12 Sixto Sánchez-Lauro Pérez, "L'Estudi General de Lleida davant un moment històric d'inflexió renovadora: peticions locals d'intervenció regi a Felip II", Revista de Dret Historic Català 9 (2009): 253-269.

13 Josep Lladonosa, Història de Lleida, vol. II (Tàrrega, F. Camps Calmet Editor, 1974), 307.

14 ACL, P7 M2 P3 C06, s.f.

15 ACL, P7_M2_P3_C04, cuadernillo, s.f. 
Miquel Despuig, doctor en ambos derechos y obispo de Lérida desde 1556, murió el 21 de noviembre de 1559 y no pudo acabar la reforma que había proyectado. Poco antes de su fallecimiento había fundado el Colegio de la Purísima Concepción y había promulgado unos estatutos para la reforma de la Universidad $^{16}$. Los paheres mandaron a Fr. Juan Moreno, catedrático de Prima de Teología, y a Melchor Munyós ${ }^{17}$, que era ciudadano honrado y paher segundo de Lérida ${ }^{18}$, a la Corte de Felipe II, a fin de obtener del monarca una sustanciosa pensión sobre la mitra de Lérida a favor del Estudio General ${ }^{19}$.

Ante la necesidad de un prelado experto para resolver la problemática del Estudio, Felipe II nombró a Antonio Agustín, que a la sazón era auditor de la Rota. El obispo, que agradeció rápidamente la merced a Felipe $\mathrm{II}^{20}$, no se dirigió directamente a Lérida, sino que encaminó sus pasos hacia Trento, donde tuvo una presencia muy activa en la última fase del Concilio ${ }^{21}$. Llegó finalmente a Lérida en 1564, e intentó aplicar allí las novedades tridentinas ${ }^{22}$.

Mientras tanto, en 1562, Felipe II había asignado 700 ducados sobre los frutos del Obispado de Lérida, a favor del Estudio General ${ }^{23}$. Estos ducados los percibía en vida el clérigo Juan Hurtado de Mendoza, y tras su fallecimiento, se acordó que Fr. Juan Moreno se trasladase a la Corte para solicitar que se hiciese efectiva la pensión de los 700 ducados a favor del Estudio, con los correspondientes abonos de los atrasos. Finalmente, el 9 de junio de 1565, el papa Pío IV concedió los 700 ducados sobre la mitra de Lérida a favor del Estudio Genera $^{24}$. Asimismo, el 28 de junio de 1568 Felipe II aprobó la unión de las rentas del Priorato de Lérida al Estudio ilerdense ${ }^{25}$.

16 Francesc Esteve Perendreu, "Rentas y reformas del Estudio General de Lérida", Analecta Sacra Tarraconensia 69 (1996): 29-86, especialmente, 69-73.

17 ACL, P7_M2_P3_C06, Llibre del Studi, f. 18r.

18 Francisco José Morales Roca, Ciudadanos y burgueses honrados habilitados como Síndicos del Brazo Real en las Cortes del Principado de Cataluña: dinastías de Trastámara y de Austria, siglos XV y XVI, 1410-1599 (Madrid: Hidalguía, 1995), 212.

19 Pedro Sanahuja, "La Universidad de Lérida y los franciscanos", Archivo Ibero-americano 7 (1947): 167-242. Véase especialmente, 210-238.

20 Constancio Gutiérrez, Trento, un problema: la última convocación del concilio (1552-1562), V, (Madrid: Universidad Pontificia de Comillas, 2000), 216-217.

21 Joan Bada i Elias, "Aportacions doctrinals d'Antoni Agustín a la tercera etapa del Concili de Trento (1562-1563)", Revista Catalana de Teologia 12/1 (1987): 125-139.

22 Juan Francisco Alcina Rovira, "Antonio Agustín y el índice de libros prohibidos del Concilio de Trento (Roma, 1564)”, Calamvs renascens: Revista de humanismo y tradición clásica 3 (2002): 7 14.

23 ACA, Cancillería, Reg. 4298, ff. 113r-v.

24 Esteve Perendreu, "Rentas y reformas...", 44-45. Véase ACA, Cancillería, Reg. 4303, ff. 115 $\mathrm{v}-116 \mathrm{v}$.

25 ACA, Cancillería, Reg. 4301, ff. 286r-287r y ACA, Cancillería, Reg. 4302, ff. 195v-196v. 
Tres meses después, Felipe II, mediante Real Cédula, le encargó a Antonio Agustín el 19 de septiembre de 1565 la reforma del Estudio General de Lérida, concediéndole amplísimos poderes para inspeccionar, sancionar y elaborar nuevos estatutos. Como veremos, dichos poderes causaron recelo por parte de las autoridades ilerdenses y crearon una situación muy problemática.

\section{LOS PROFESORES DEL ESTUDIO}

Gracias a unas notas de pagos, contenidas en el Llibre del Studi que se conserva en el Archivo Capitular de Lérida tenemos una documentación inédita acerca del profesorado del Estudio General cuando llegó el obispo Antonio Agustín, y que complementan el trabajo que hizo Pedro Sanahuja sobre las ápocas de pago anteriores ${ }^{26}$.

Hay que indicar, antes de entrar en la nómina de lectores, que en el manuscrito se mezclan las cátedras doctorales con las de bachiller. Esta distinción sólo es importante para los juristas, ya que había tres cátedras de leyes y tres de cánones, cuatro catedrillas de leyes y cuatro de cánones, así como una cátedra de instituta, que podía leer tanto un bachiller como un doctor. Había asimismo cuatro cátedras de teología, tres de medicina, dos de filosofía y dos de gramática. Estas cátedras, como veremos, quedaron definitivamente asentadas tras la reforma de Antonio Agustín de 1575.

Para el curso de 1564, las notas de pagos proporcionan los siguientes nombres de lectores, que hemos reunido aquíi ${ }^{27}$ : Pere Molí, Pere Segarra, Bartomeu Gomar, Pere Rubio (Pere Aguiló, sustituto), Francesc Botella, Hieronim Pasqual, Agustí Gascón, Gabriel Babot, Francesc Riber, Fernando Gil Scalona, Jaume Mir, Antoni Oliba, Luis Copons, Llorens Cellers, Andreu Rius, Salvador Mor, Miquel Ferrer, Fra Joan Ginebrosa, Mestre Pere Llopico, Thomas Carrera, Barthomeu Serret y Pere Esteve ${ }^{28}$.

Hay que recordar, en primer lugar, que el catedrático de Prima de Teología, Fr. Juan Moreno, que leía -según Sanahuja y Vázquez Janeiro desde $1549^{29}$ - se

26 Sanahuja, "La Universidad de Lérida y los franciscanos", 204-205.

27 Mantenemos la grafía original, que sólo alteramos en contados casos en la que es necesario hacerlo para que resulte comprensible. Las anotaciones entre paréntesis son aclaraciones que aparecen en el propio texto manuscrito.

28 ACL, Llibre del Studi, ff. 27r-28r.

29 Isaac Vázquez Janeiro, "Repertorio de franciscanos españoles graduados en teología durante la edad media", en Repertorio de Historia de las Ciencias Eclesiásticas de España, vol. 3: Siglos XIII- XVI (Salamanca: UPSA, 1971), 282. 
hallaba en la Corte para negociar las rentas de la Universidad. Moreno era franciscano conventual, nacido en Monzón ${ }^{30}$, había leído durante muchos años en Lérida y a la sazón se desempeñaba como confesor de Isabel de Valois. De ahí que no conste su sueldo hasta 1568 , año de la muerte de la reina. Desde que los conventuales ilerdenses tuvieron que dejar su cenobio a los observantes, Sanahuja no tenía más noticias de Moreno $^{31}$. Gracias a la documentación que aquí transcribimos podemos saber que este religioso siguió como catedrático durante algunos años más, como veremos a continuación.

Pere Molí, Pere Segarra y Bartomeu Gomar ${ }^{32}$ eran doctores en derecho canónico y catedráticos de dicha Facultad, mientras que Pere Rubión ${ }^{33}$, Francesc Botella $^{34}$, Hieronim Pasqual eran doctores y catedráticos de Leyes, que ya estuvieron presentes en la reforma de los Estatutos de la Universidad realizada por el obispo Despuig en $1559^{35}$. Agustí Gascó era doctor y catedrático de Teo$\operatorname{logía}^{36}$. Gabriel Babot era catedrático de Medicina, al igual que Gil Escalona ${ }^{37}$, que leyó muchos años en la Facultad.

Antoni Olibà estudió en Lérida, era catedrático de Leyes como mínimo desde ese $\operatorname{curso}^{38}$ y fue un destacado jurisconsulto, como después veremos. Andreu Rius fue insaculado como oidor y diputado real, años después en calidad de doctor en leyes por la ciudad de Tortosa ${ }^{39}$, mientras que Tomás Carrera lo fue por la ciudad de Barcelona ${ }^{40}$. Hallamos también como joven profesor a Lluís de Copons, quien con el tiempo llegó a ser Abogado Fiscal de la Generalitat ${ }^{41}$. Fr. Joan Ginebrosa era un dominico, bachiller en Artes por la Universidad de Barcelona $^{42}$, destinado en aquel momento para enseñar Artes en Lérida. Indi-

30 Félix de Latassa, Biblioteca Nueva de los escritores aragoneses: que florecieron desde el año de 1500 hasta 1599, Vol. 1 (Pamplona, en la oficina de Joaquín Domingo, 1798), 309-311.

31 Sanahuja, "La Universidad de Lérida y los franciscanos", 238.

32 Insculado como oidor real, Eva Serra i Puig, Els Llibres de l'Ànima de la Diputació del General de Catalunya (1493-1714), II (Barcelona: IEC, 2015), 198.

33 Insaculado como oidor y diputado real, Serra i Puig, Els Llibres de l'Ànima..., 379.

34 Insaculado como oidor y diputado real, Serra i Puig, Els Llibres de l'Ànima..., 67.

35 Esteve Perendreu, El Col·legi universitari de l'Assumpció..., 58.

36 Francesc Esteve Perendreu: "La docència de la teologia a Lleida, la càtedra del bisbe Conchillos i les altres càtedres teològiques de 1'Estudi General" en X. Company (ed.), El bisbe Jaume Conchillos, l'humanisme a Catalunya (Lleida: Amics de la Seu Vella, 1993), 159.

37 Josep Lladonosa, La Facultat de Medicina de l'antiga Universitat de Lleida (Lleida: Rafael Dalmau, 1969), 46.

38 Esteve Perendreu, El Col·legi universitari de l'Assumpció..., 58.

39 Insaculado como oidor y diputado real, Serra i Puig, Els Llibres de l'Anima..., 364.

40 Insaculado como diputado real, Serra i Puig, Els Llibres de l'Ànima..., 94.

41 Miguel Ángel Martínez Rodríguez, "Linaje y poder en la Cataluña foral: la actividad política de los Copons", Cuadernos de Historia Moderna 22 (1999), 11-31, especialmente, 18. 15

42 Josep M. Madurell Marimon, "Luis Juan Vileta”, Analecta Sacra Tarraconensia 37 (1964), 
quemos que Bartomeu Serret era canónigo de Lérida ${ }^{43}$ y profesor de cánones, $\mathrm{y}$ que también enseñaba derecho canónico Pere Esteve.

En 1565 la nómina de profesores estaba compuesta por Pere Molí, Pere Segarra, Bartomeu Gomar, Pere Rubio (Pere Aguiló, sustituto), Francesc Botella, Hieronim Pasqual, Agustí Gascó, Pere Salas, Gabriel Babot, Francesc Riber, Fernando Gil Scalona, Jaume Mir, Antoni Oliba, Joan Garcia, Llorens Cellers, Barthomeu Serret, Andreu Riu, Salvador Mor, Thomas Carrera, Miquel Ferrer, Joan Ginebrosa, Pere Llopico, Pere Esteve y el "pare Boneta, lector d'Arts ${ }^{44 "}$.

Este último era el mercedario Jerónimo Boneta, que enseñó Artes en Lérida y fue catedrático de Teología en la Universidad de Huesca entre 1578 y $1585^{45}$. De Pere Salas podemos decir que fue doctor en Teología y rector de la Iglesia parroquial de Sant Joan de la Plaça ${ }^{46}$, así como también examinador de libros para el Santo Oficio ${ }^{47}$. A continuación, en el manuscrito se indica la nómina de los bachilleres en leyes y cánones:

"Mes avant se continuen asi los bachillers ordinaris qui llegiran per la conducta tant en canons com en lleys, los quals han de llegir quaranta lliçons qui son en dies legibles a raho tres sos per lliçons, los quals lectors son los seguents y es la lectura del primer any

Primo $\mathrm{M}^{\mathrm{o}}$ Barthomeu Serret. Andreu Rius, Llorenç Sellers, Salvador Mor, Jaume Mir, Antoni Oliba, Thomas Carrera, Joan Garcia ${ }^{48}$ ".

Nuevos detalles aparecen en la nómina de lectores de 1566: Pere Molí, Pere Sagarra, Bartomeu Gomar, Francesc Botella, Pere Aguiló, Francesc Bonet, Miquel Montornés, Gabriel Babot, Francesc Riber, Jaume Mir, Antoni Oliba, Francesc de Sinistere i Francesc Bafart, Llorenç Sellers, Bartomeu Serret, Pere Esteva, Salvador Mor, Tomas Carrera, Jeroni Boneta, Bartomeu Vilanova (lectura de Artes), Joan Sanz, Gabriel Maçot (Gramática) ${ }^{49}$.

43 Ramiro Viola i González, "Un testament canonical (16 d'agost de 1561)”, Analecta Sacra Tarraconensia 71 (1998), 915.

44 ACL, Llibre del Studi, ff. 29r-30r.

45 Antonio Durán Gudiol, "Notas para la historia de la Universidad de Huesca en el siglo XVI," Hispania Sacra 21 (1968): 87-150. Véase especialmente, 150.

46 Esteve Perendreu, "La docència de la teologia...", 199.

47 [Mariano Aguiló], Catálogo de obras en lengua catalana impresas desde 1474 hasta el presente (s.1: 1865), 168.

48 ACL, Llibre del Studi, f. 30v.

49 ACL, Llibre del Studi, ff. 33r-34r. 
En primer lugar hay que destacar que Pere Aguiló ${ }^{50}$ consolidó la cátedra de segunda de Leyes y que Francesc Bonet ${ }^{51}$ pasó a ser catedrático en lugar de Jeroni Pasqual. Tenemos una breve noticia de Miguel Montornés, que después fue presbítero y vicario de Leciñena, y que fue acuchillado en 1572 por Antonio Clavero, agricultor y habitante en dicha localidad ${ }^{52}$. Aparece ya en esta nómina Francesc Bafart, colegial y prior del Colegio de la Asunción ${ }^{53}$, que a la sazón era lector de leyes. Hay que destacar que Bartomeu Vilanova era un reputado estudioso de Aristóteles, que había enseñado previamente en la Universidad de Valencia y que, tras su paso por Lérida, lo hizo en la de Barcelona ${ }^{54}$. Hasta ahora no se sabía qué años había leído Vilanova en Lérida, aunque era considerado por la historiografía uno de los metafísicos más notables de la Corona de Aragón ${ }^{55}$.

En cuanto a los lectores de 1567, la documentación manuscrita proporciona los siguientes nombres: "Joan Moreno y a ell mestre Jaume Come lector de taulogia", Agustí Gascón, que leía la cátedra de Conchillos, Francesc Berenguer, Pere Salas, Pere Molí (lector en cánones), Pere Sagarra, Bartomeu Gomar, Salvador Mor (bachiller), Bartomeu Serrat, Llorenç Cellers, Pere Esteve, Botella (lector de leyes), Pere Aguiló, Micer Bonet, Antoni Oliba (bachiller), Jaume Mir, Thomas Carrera, Baffart, Gabriel Babot (lector en medicina), Francesc Riber, Fernando Scalona, Miquel Llupia (para leer las faltas de los otros doctores de la Facultad de Medicina), Bartomeu Vilanova (lector en Artes), Joan Sans, Celedoni Prunyonosa (lector de gramática) y Antoni Seran (ayudante de Prunyonosa) ${ }^{56}$.

Indiquemos que Francesc de la Torre Berenguer era canónigo de la catedral de Lérida y catedrático de Teología, mientras que Pedro Salas era doctor en Teología y catedrático de dicha disciplina. En 1573 eran, respectivamente, catedráticos de prima y de segunda de Teología, tal y como consta en la aprobación

50 Insaculado como diputado real, vid. Serra i Puig, Els Llibres de l'Ànima ..., 10.

51 Insaculado como oidor real, vid. Serra i Puig, Els Llibres de l'Ànima..., 62.

52 Se abrió un proceso penal. Véase Maria Isabel Falcón Pérez, Miguel Ángel Motis Dolader, Procesos criminales en el Arzobispado de Zaragoza (Zaragoza: Gobierno de Aragón, 2000), 115.

53 Esteve Perendreu, El Col'legi universitari de l'Assumpció..., 57.

54 Fernández Luzón, La Universidad de Barcelona ..., 155, 314-315.

55 Jordán Gallego Salvadores, "La metafísica en España durante el siglo XVI", en Repertorio de las ciencias eclesiásticas en España, VII (Salamanca: UPSA, 1979), 229-230.

56 ACL, Llibre del Studi, ff. 37r-v. 
del Libro llamado Consuelo de Nuestra Peregrinación de Fray Andrés Capi$11 a^{57}$.

En 1568 hubo los siguientes lectores sustitutos: Miquel Tristany (sustituto de Molí), a quien no se le debía nada, porque en realidad no había leído ("nihil, quia non legit"); Pere Bordalba (sustituto de Micer Sagarra); Miquel Ribes (sustituto de Gomar); Tomas Carrera (sustituto en leyes); Antoni Benach (sustituto del maestro Babot); Miquel Llupia (sustituto del maestro Riber); Lluys Fuster (bachiller sustituto en cánones); Melchor Punster (sustituto en leyes); Rafael Monço y Josep Calaf (sustitutos en leyes) ${ }^{58}$. Recordemos que Miquel Tristany, doctor en derechos, fue conservador del Estudio General en junio de $1568^{59}$ y que los demás, mediado el tiempo, devinieron catedráticos del Estudio General.

En el curso 1568-1569 encontramos los siguientes lectores: Joan Moreno, Agustí Gascó (Conchillos), Francesc Berenguer, Pere Salas, Pere Molí (lector de cánones), Pere Segarra, Bartomeu Gomar, Francesc Gomar (lector de leyes), Pere Aguiló, Francesc Bonet, Pere Joan Benach (lector de medicina), Francesc Riber, Ferrando Gil de Ascalona, Joan Sans, Fra Boneta, Fr. Francesc Salvador (que leía las horas del maestro Vilanova), Pere Joan Nunyes, Pere Bordalba (sustituto de Pere Sagarra), Miquel Ribes (sustituto de Bartomeu Gomar, al cual no se le pagó nada, porque no leyó), Pere Estamper, Jaume Roig, Jaume Calbis, Gaspar Punster, Tomas Carrera, Francesc Bafart, Joan Mur, Melchor Punster, Luis Fuster (sustituto en cánones), Rafel Montsó (sustituto en leyes del maestro Carrera), Francesc Descamps (sustituto en leyes del maestro Carrera) y Pere Plana (sustituto de Baffart) ${ }^{60}$.

Sabemos que Joan Mur fue colegial de la Asunción y Rector del Estudio durante el curso de 1567-1568 y Pere Plana era también colegial de la Asunción $^{61}$. Con todo, el profesor más interesante, desde el prisma historiográfico, es Pere Joan Nunyes (1522-1602). Sin duda, Nunyes, seguidor de Ramus $^{62}$, era uno de los humanistas hispanos más prestigiosos a la sazón y, al parecer, tras haber impartido clases en diferentes universidades, encontró protección

57 Andrés Capilla, Libro llamado Consuelo de Nuestra Peregrinación de grande utilidad y consolaciòn para todos los fieles, en que se trata de la dignidad y exellencia de la Religión Christiana (Lerida: Pedro de Nobles y Juan de Villanueva, 1574).

58 ACL, Llibre del Studi, ff. 41v-42v.

59 Esteve Perendreu, Mestrescoles i rectors..., 32.

60 ACL, Llibre del Studi, ff. 46r-47r.

61 Esteve Perendreu, El Col-legi universitari de l'Assumpció..., 58.

62 Juan Francisco Alcina Rovira, "Los inicios del ramismo en España", en J. Pérez Dura y J. M. Estellés, (eds.), Los humanistas valencianos y sus relaciones con Europa (Valencia: Ajuntament de Valencia, 1998), 117-136. 
en casa de su amigo Antonio Agustín. Procedente de la Universidad de Zaragoza, indica Pilar Barbeito que "lo cierto es que en 1568 lo encontramos en Lérida, en casa de Antonio Agustín, obispo de la diócesis desde 1561, con quien le unía una buena amistad que no hizo sino estrecharse con los años ${ }^{63}$ ".

En la tesis doctoral de Ferran Grau ${ }^{64}$ se sugería que Nunyes probablemente tuvo relación con el Estudio General ilerdense. Gracias al manuscrito del que damos cuenta sabemos que Nunyes enseñó Artes durante varios cursos en Lérida. Indicaba Pilar Barbeito que "tras estas noticias de la estancia en Lérida de 1568 se pierde de nuevo la pista de Núñez, aunque es probable que entre 1568 y 1574 -año en que lo encontramos de nuevo en Zaragoza- pasara algún periodo en la Universidad de Barcelona ${ }^{65}$ ". Antonio Fernández Luzón indica en la nómina de profesores del Estudio barcelonés que Nunyes enseñó retórica y griego desde el curso 1572-1573 hasta 1579-1580 ${ }^{66}$.

La documentación del Estudio de Lérida concuerda completamente, pues como veremos- hemos documentado allí a Nunyes hasta el año 1571. No hay duda de que el humanista fue a la ciudad del Segre atraído por la amistad de Antonio Agustín, que le hospedó y le dejó cultivarse y recrearse en su biblioteca personal. Hay que conjeturar que la creciente presión de los paheres hacia el obispo y el carácter sensible de Nunyes motivaron su traslado a la Universidad de Barcelona que, como veremos, acogió a muchos profesores ilerdenses en esta década de 1570, especialmente tras algunos incidentes entre el Rector, los Paheres y el Obispo.

Pasemos a detallar la nómina de los lectores ordinarios que percibieron la paga de San Juan de 1570: Joan Moreno, Francesc de la Torre alias Berenguer, Pere Sales (cátedra de Conchillos), Joan Tarros, Gaspar Sahona, Pere Moli, Pere Segarra, Bartomeu Gomar, Francesc Botella, Pere Aguiló, Francesc Benet, Antonio Joan Benach, Francesc Riber, Ferrando Gil de Escalona, Joan Sans (Artes), Pere Joan Nunyez (Artes), Pere Joan Nunyez (lectura de Gramática), P. Salvador (lectura de Artes), Pere Esteve (lector en cánones), Gaspar Punster, Antoni Oliba, Tomas Carrera, Francesc Baffart, Melchior Punster, Lluis Fuster (que ha leído por Jaume Roig) y Miquel Ferrer (por el resto de las lecturas) ${ }^{67}$.

63 M. Pilar Barbeito Díez, Pedro Juan Núñez, humanista valenciano, tesis doctoral (Madrid: Universidad Complutense, 1996), 57.

64 Ferran Grau, Las Retóricas de Pedro Juan Núñez, tesis doctoral (Valencia: Universidad de Valencia, 1994), LXXIII-LXXV.

65 Barbeito Díez, Pedro Juan Núñez..., 60.

66 Fernández Luzón, La Universidad de Barcelona..., 311.

67 ACL, Llibre del Studi, ff. 55r-56r. 
De estos profesores, cabe destacar especialmente a Joan Tarròs, a quien identificamos con el futuro Abad de Poblet (1598-1602). De él sabemos que fue Rector del Colegio de Poblet en Lérida en 1570 y Maestro en Sagrada Teología $^{68}$. También sobresalió Fr. Gaspar Sahona (o Saona), natural de Mota del Cuervo, Maestro en Sagrada Teología y más adelante Provincial de la Orden de San Agustín en la Provincia de la Corona de Aragón ${ }^{69}$.

Detengámonos también en la figura de Miquel Ferrer, cuyo nombre ha aparecido ya antes. Aparece aquí como profesor del resto de lecturas y la razón es que daba clase tanto de Artes como de Medicina. Fue autor de una Recollectio casuum. Mètodo y art molt clar pera apendrer la gramàtica de la llengua llati$n a$, publicada en Lérida en $1578^{70}$. En una carta al obispo Antonio Agustín le exponía su plan de formación humanística dirigida a los discípulos ilerdeneses ${ }^{71}$.

En la nómina de profesores en la festividad de San Juan de 1571 aparecen: Joan Moreno, Francesc de la Torre, Pere Salas, Antoni Pont, Joan Tarros, Gaspar Saona, Pere Moli, Pere Sagarra, Bartomeu Gomar, Botella, Pere Aguiló, Bonet, Fernando Gil de Escalona, Pere Arnau de Sant Marti, Joan Sans, Pere Joan Nunyez, Montserrat Capdevila, Gabriel Massot (leyó la gramática de Nunyes), Pere Bordalba, Miquel Ribes, Tomas Carrera, Melchior Punster, Francesc Boldu (sustituto de Riber), Miquel Ferrer (sustituto de Scalona), Agusti Calaf (sustituto de Sant Marti), Matheu Pla, Lluis Fuster, Joseph Calaf, Cristofol Capero, Antoni Oliba, Rafael Monço, Pere Plana, Francesc Moliner, Miquel Ferrer, Josep Morata (como sustituto de Joseph Calaf en cánones) ${ }^{72}$.

Por un lado, creemos que el profesor Antoni Pont fue el célebre trinitario mallorquín, estudioso de la obra de Ramon Llull ${ }^{73}$. Al haber sido profesor en el Estudio de Lérida durante su juventud, y al tenerlo localizado en Lérida desde 1567 hasta 1577 nos parece probable que se trate del mismo. Por otro lado, Agustí Calaf fue doctor en leyes ${ }^{74}$ y catedrático del Estudio General e hijo de un

68 Jaime Finestres y de Monsalvo, Historia del Real Monasterio de Poblet, vol. 4 (Tarragona: Joseph Barber, 1756), 220.

69 José Massot, Compendio Historial de los Hermitaños de nuestro Padre San Agustín del Principado de Cataluña (Barcelona: Juan Jolis, 1699), 71-73.

70 Lladonosa, "Humanisme i Reformes...", 95.

71 Esteve Perendreu, Mestrescoles i rectors..., 152.

72 ACL, Llibre del Studi, ff. 62r-63r.

73 Joaquín M. Bover, Biblioteca de autores baleares, vol. 2 (Palma: Gelabert, 1868), 129. Oriundo de Selva (Mallorca), Antoni Pont nació hacia 1500 tomó el hábito trinitario en 1518 y fue a estudiar a Lérida, donde fue maestro y lector -al decir de Bover y otros-por espacio de veintidós años. Regresó en 1553 y de allí fue destinado a Murviedro, para pasar a Lérida como ministro en 1567 hasta que en 1577 tuvo la misma dignidad en Mallorca. Felipe II promovió su elección como arzobispo de Orestano, a la que renunció. Murió en Palma en 1580.

74 DGC, Vol. 3, p. 126. 
homónimo ciudadano honrado y paer de Lérida ${ }^{75}$. Francesc Moliner fue jurista, insaculado después como diputado militar en la veguería de Lérida en lugar del ya citado Jeroni Botella ${ }^{76}$.

Tenemos también los salarios de los lectores en la Festividad de San Juan de 1572. La nómina esta compuesta por Joan Moreno, Francesc de la Torre, Pere Salas (cátedra del obispo Conchillos), Antoni Pont, Joan Tarros, Gaspar Sahona, Pere Moli, Pere Sagarra, Bartomeu Gomar, Francesc Botella, Pere Aguiló, Francesc Bonet, Francesc Riber, Fernando Escalona, Pere Arnau Sant Marti, Montserrat Capdevila (curso), Nofre Serra (curso), Siurana (lectura de gramática), Pere Bordalba (sustituto de Molí), Miquel Ribes (sustituto de Segarra), Serret (sustituto de Gomar), Tomas Carrera (sustituto de Botella), Melchior Punster (sustituto de Aguiló), Miquel Ferrer (sustituto de Riber); Lluis Fuster, Cristofol Capero, Pere Mollan, Rafel Monço, Pere Plana, Francesc Moli, Jeronim Pradell (sustituto de Fuster), Josep Morata (sustituto de Capero), Francesc Tormo (sustituto de Saona), Josep Coria (sustituto de Oliba), Miquel Berart (sustituto), Joan Crespo (sustituto), Pere Montserrat (sustituto), Luis Sans (sustituto $)^{77}$.

Resulta llamativo el caso de Nofre Serra. Recordemos que hasta el curso anterior había leído Nunyes. ¿Podría ser este Nofre Serra el célebre Onofre Serra, profesor de Artes en la Universidad de Valencia? No pasa de ser una conjetura: sabemos que el Patriarca Ribera había intervenido duramente en la Universidad de Valencia y había encarcelado en la prisión eclesiástica a varios catedráticos (entre los que se hallaba Serra), que fueron liberados en 1571, si bien el proceso inquisitorial se prolongó hasta febrero de $1572^{78}$. ¿Es posible que Serra hubiera pasado a Lérida huyendo de estos problemas, buscando un ambiente humanístico menos enconado? Otro nombre llamativo es el de Vicent Ciurana, que identificamos con el homónomo gramático y erudito valenciano ${ }^{79}$.

Aparece en esta nómina por primera vez el arcediano Pere Mollan, destacado humanista, gran amigo de Antonio Agustín y síndico suyo en Roma, citado en su correspondencia en temas de erudición jurídica ${ }^{80}$ y también en otras

75 Morales Roca, Ciudadanos y burgueses honrados..., 88.

76 Serra i Puig, Els Llibres de l'Ànima..., I, 170.

77 ACL, Llibre del Studi, ff. 71r-73r.

78 Amparo Felipo Orts, La Universidad de Valencia durante el siglo XVI (1499-1611), (Valencia: Universidad de Valencia, 1993), 45.

79 Justo Pastor Fuster, Biblioteca Valenciana de los escritores que florecieron hasta nuestros dias, vol. 1, (Valencia: Ximeno, 1827), 189.

80 Antonii Augustini archiepiscopi Tarraconensis Opera omnia, Vol. 7 (Lucae: Typis Josephi Rochii, 1772), 229. 
cuestiones prosaicas (más bien de carácter pecuniario). Mollán era también valenciano y fue albacea testamentario de Pedro Chacón ${ }^{81}$.

Desde el punto de vista del saber jurídico, el personaje más interesante es el Dr. Francesc Molí, hijo del ya citado Pere Molí. Tras haber sido profesor en la Universidad de Lérida fue nombrado oidor en la Real Audiencia de Mallorca $^{82}$. Posteriormente regresó a Lérida, donde volvió a enseñar. Fue autor de varios libros sobre derecho civil y canónico (sobre los pactos matrimoniales, y sobre las juridicciones secular y eclesiástica ${ }^{83}$. Creemos asimismo que el citado Joan Crespo fue, mediado el tiempo, el homónimo oidor de la Audiencia de Mallorca, que falleció al poco de tomar posesión de su plaza ${ }^{84}$.

Es posible que Pere Montserrat, doctor en derecho, fuese el diputado insaculado por Barcelona en 1581 y que aparece como fallecido en $1588^{85}$. En cuanto a Lluís Sans, cabe examinar la posibilidad de que fuese el futuro canónigo de la Seu d'Urgell, obispo electo de Elna, primer obispo de Solsona y posteriormente obispo de Barcelona. Sabemos que se doctoró en ambos derechos en Roma el 30 de abril de $1576^{86}$.

La última nómina completa que tenemos es la de San Juan de 1573. En ella hallamos a los siguientes lectores: Joan Moreno, Francesc de la Torre, Pere Sales, Antoni Pont, Joan Terros, Gaspar Assahona, Barthomeu Gomar, Pere Bordalba, Bartomeu Serret, Francesc Botella, Pere Aguiló, Francesc Bonet, Francesc Riber, Fernando Gil de Escalona, Pere Arnau de Sant Marti, Onofre Serra, Ramon Cirera, Antoni Mani, Vicent Ciurana (maestro de gramática), Miquel Ferrer (maestro de gramática), el maestro Armeda (sustituto del maestro Berenguer), Francesc Pastor (sustituto del maestro Aguiló por enfermedad),

81 Elisa Ruiz, "Los años romanos de Pedro Chacón”, Cuadernos de Filología Clásica 10 (1976): 189-247, especialmente, 209.

82 Antonio Planas Rosselló, La Real Audiencia de Mallorca en la época de los Austrias (15711715), (Barcelona: Publicacions de la Universitat Pompeu Fabra, 2010), 320, aporta los siguientes datos: "Nombrado oidor de Mallorca el 16 de enero de 1585. Realizó sendas visitas a los oficiales e instituciones de la isla de Menorca en 1587-1588 y 1596-1597. Fue suspendido del cargo en 1597 como consecuencia de un procedimiento de visita en el que se le imputaron los delitos de concubinato, violación y estupro de menores. En julio de aquel año partió haciala Corte para defenderse. Fue privado de la plaza y condenado a una multa de 1.500 ducados".

83 Francisci Molini, De brachio seculari Ecclesiae praestando et mutuis iudicum auxiliis celeberrimi commentarij: in tres libros distincti; his accessit liber singularis De sacra homicidio amittenda immunitate nec ne (Barcelona: Ioannem Simon, 1607); Tractatus celebris, et insignis de ritu nuptiarum, et pactis in matrimonio conuentis (Barcelona: Laurentij Déu, 1617).

84 Planas Rosselló, La Real Audiencia de Mallorca..., 312.

85 Serra i Puig, Els Llibres de l'Ànima ..., I, 254.

86 Rafael Ramis Barceló, Doctores hispanos en Leyes y Cánones por la Universidad de la Sapienza de Roma (1549-1774) (Madrid: Dykinson, 2017), 73. 
Pere Esteve (sustituto del maestro Bordalba), Tomas de la Carrera (sustituto del maestro Botella), Lluis Fuster (ordinario en cánones), Joseph Calaf (enfermo), Cristofol Capero, Pere Mollan, Antoni de Oliba, Pere Plana, Francesc Moli, Joan Crespo, Lluis Sans, Josep Morata, Francesc Tormo, Jeroni Pintor (sustituto), Pere Monserrat (sustituto), Joan Castello (sustituto), Joan Baptista Boyl (sustituto), Lois Vilallonga (sustituto) ${ }^{87}$.

Más allá de los maestros que ya hemos comentado, hay que mencionar a Ramon Cirera, un autor que nos permite abundar en la conexión valenciana, pues hallamos a un personaje homónimo en la colación de grados en la Universidad de Valencia, junto al ya citado Onofre Serra, en el lustro de 1566$1571^{88}$. En cuanto a Antoni Maní, beneficiado de la catedral, hay que suponer que a la sazón leía Artes o Filosofía. Desde 1578 detentó una cátedra de teología y más adelante fue canónigo penitenciario, hasta su muerte acaecida en $1610^{89}$.

Hay que descartar, por razones cronológicas que este Francesc Pastor fuese el futuro maestrescuela del Estudio. En lo tocante a Joan Baptista Boil fue, como veremos, Rector del Estudio durante el curso 1574-1575. Y en cuanto a Joan Castelló, fue un destacado jurista, más tarde profesor en la Universidad de Barcelona y que llegó a ser juez de la Real Audiencia ${ }^{90}$. Participaba de los ideales humanistas y su biblioteca estaba bien surtida con las obras de Tito Livio, Cicerón, Virgilio, Herodoto... ${ }^{11}$

A partir de 1573 dejamos de tener datos del profesorado de Lérida. Desde entonces, como veremos, se puso de manifiesto una gravísima tensión entre el Estudio, los paheres y el obispo. Lo cierto es que, en paralelo a la gestación de este conflicto, en 1573 empezó un éxodo hacia la Universidad de Barcelona de un grupo muy representativo de estos profesores.

87 ACL, Llibre del Studi, ff. 80r-73r.

88 Amparo Felipo Orts, Francisco J. Peris Felipo, Francisca Miralles Vives, Estudiantes y probanzas de cursos en la Universidad de València (1561-1707) (Valencia: Universidad de Valencia, 2013) 411 .

89 Esteve Perendreu, Mestrescoles i rectors..., 155.

90 Fernández Luzón, La Universidad de Barcelona..., 277.

91 Carme Narváez Cases, "El patronatge de les noves oligarquies urbanes a l'art català dels segles XVI i XVII", Recerques 51 (2005): 18. 


\section{EL CONFLICTO SOBRE LA REFORMA DE LA UNIVERSIDAD (1572-} 1575)

Pese a que Antonio Agustín había recibido el encargo específico de reformar la Universidad, lo cierto es que las medidas concretas no llegaban. Buena parte de la historiografía (Villanueva ${ }^{92}$, Sainz de Baranda ${ }^{93}$, Poch $^{94}$, Esteve Perendreu ${ }^{95}$ ) achaca el enfado de los Paheres a la poca celeridad del obispo, mientras que Lladonosa ${ }^{96}$ considera que hubo desencuentros personales, incomprensión y falta de entendimiento, que desembocaron en una situación muy tensa.

Pese a que los incidentes de los últimos años del pontificado hayan sido ya tratados por los autores mencionados, queremos aportar aquí nueva documentación, que tal vez sirva para esclarecer un poco más la situación. Más allá de los documentos que fue transcribiendo y publicando José Poch, hay que tener en cuenta la riqueza de la correspondencia que se ha conservado de los Paheres, que dan noticias indirectas del conflicto.

Ya el 16 de julio de 1568, Felipe II había insistido en que se llevase a cabo la reforma definitiva del Estudio. Pese a las reiteradas peticiones de los Paheres y del Rector, el obispo no había hecho ninguna intervención decisiva, aunque había acogido a diversos profesores humanistas y les había facilitado su estadía en Lérida. El Dr. Francesc Molí fue enviado a la corte con una letra de presentación el 27 de marzo de 1572 para el "redreç" (enderezamiento) de la Universidad $^{97}$.

La tensión llegó al cénit en abril de 1573 cuando se eligió como Rector del Estudio al bachiller Gil Gironza, y poco después se llevó a cabo otra elección que recayó en Gaspar Beranuy. Los Paheres entiendieron que la primera se había hecho de acuerdo con los privilegios y estatutos del Estudio, mientras que el obispo visitador-reformador consideró que los privilegios se habían observado

92 Jaime Villanueva, Viage literario a las iglesias de España, T. XVII (Madrid: Imprenta de la Real Academia de la Historia, 1851), 56-58.

93 Pedro Sainz de Baranda, España Sagrada, T. XLVII (Madrid: Imprenta de la Real Academia de la Historia, 1850), 93.

94 José Poch, "Del Estudio General de la Universidad de Lérida. Carta de los Paheres a Felipe 11 (17 agosto 1574)", Ciudad, 26 (1974): 120; José Poch, "El rectorado del Estudio General de Lérida en la anualidad 1573-1574", Ciudad, 27 (1975): s.n.

95 Esteve Perendreu, "Rentas y reformas...", 39-51.

96 Lladonosa, "Humanisme i Reformes...", 92.

97 AMP, Reg. 850 (1572-1574), f. 56r. 
solamente en la segunda elección. Las autoridades confirieron el Rectorado a Gironza, mientras que el obispo sólo reconocía a Beranuy ${ }^{98}$.

La decisión del obispo fue impugnada por los Paheres ante el virrey de Cataluña, Fernando de Toledo, manifestando que no se le diese posesión a Gaspar Beranuy, porque era una persona muy violenta y conflictiva. Escribieron los Paheres al virrey el 28 de abril de 1573, indicando que "dit Beranuy ha comensat havent manasat pegar unes bastonades a un estudiant dels electors de dit rector de la ciutat de Barbastre anomenat Leonardo" 99 . El asunto llegó a la Real Audiencia.

Mientras tanto, el embajador de Lérida, "por ser los salarios que estan señalados para los cathedraticos pequeños" y porque tenían miedo de que se fuesen a otras universidades y no se encontrarían personas adecuadas para leer, pedía al Rey un aumento de las Rentas del Estudio. Felipe II respondió el 21 de mayo de dicho año, ordenando que si quedaban rentas vacantes se diesen a la Universidad Ilerdense ${ }^{100}$.

La Audiencia de Barcelona falló el 22 de mayo, confirmando la decisión del obispo visitador-reformador sobre el Rector. Los paheres volvieron a escribir al Virrey el 10 de junio indicando que, sobre el caso de Beranuy, "per ser persona tan escandalosa", habían pedido consejo al Dr. Gomar, lector de Prima $^{101}$. Con todo, al final, el 10 de noviembre el Virrey ratificó la decisión de la Real Audiencia.

Esta decisión, lejos de calmar los ánimos, los encendió aún más. Juan Bautista Bohil fue elegido rector para el curso 1574-1575 y tomó posesión el primer día de marzo. Como el obispo no llevaba a cabo la reforma, los Paheres, a la vista de los problemas, decidieron escribir directamente a Felipe II. En su carta de 17 de agosto indicaban que:

“...vista la gran ruhina y total perdicio causades en la Universitat del Studi per lo Reverent pare en Crist bisbe de aquesta ciutat havent nos portat sembre ab rahons bones sperant y nou o deu anys que ha que te lo carrech y V. M. de visitador y reformador de dita Universitat reformes aquella haventlo y infinides voltes pregat y tambe donada la carta de V. Majestat de 16 de juliol de 1568 ab

98 Esteve Perendreu, "Rentas y reformas...," 49.

99 AMP, Reg. 850 (1572-1574), f. 62r.

100 ACA, Cancillería, Reg. 4305, ff. 94v-95r.

101 AMP, Reg. 850 (1572-1574), f. 64r. 
la qual fent nos la mentres ei manave fes dita reformacio dins lo termini de sis $\operatorname{mesos}^{102}$ ".

Los Paheres se quejaban también de que el obispo no había llegado a hacer la ansiada reforma y que cada vez había menos estudiantes. Pedían también al Rey que las cátedras se diesen por votos. Esta misma idea fue repetida en tres cartas enviadas el mismo día a Pau Pla (abad de Sant Pere de Galligants, canónigo de la Catedral de Barcelona y doctor del Real Consejo Civil), al vicecanciller Bernardo de Bolea y al abogado fiscal Miquel Terça ${ }^{103}$.

A principios de septiembre, el obispo, deslegitimando a Bohil como Rector $^{104}$, mandó a algunos hombres armados para que lo capturaran y le quitaran las insignias rectorales ${ }^{105}$. Los Paheres escribieron a Fernando de Toledo el 4 de septiembre para referirle los hechos y también a Miquel Revert, síndico de la Ciudad de Lérida, indicándole que el Rector iba al Estudio para la colación de un grado y que el obispo mandó "a uns criats armats ab penyals ${ }^{106 ", ~ l e ~ q u i-~}$ taron las insignias rectorales y se lo llevaron preso al Palacio Episcopal. De inmediato, mucha gente de la ciudad la emprendió contra el obispo y éste, temiendo una rebelión, dejó salir libre al Rector.

Los Paheres escribieron el 8 de septiembre al virrey Fernando de Toledo para que intercediese ante el rey a fin de lograr la reforma de la Universidad, indicándole que el obispo había puesto en libertad al Rector ${ }^{107}$. El 23 del mismo mes escribieron al Rey para pedir de nuevo el "redreç" de la Universidad ${ }^{108}$.

En una nueva misiva de los Paheres al monarca, fechada el 7 de octubre de 1574, se quejaban de que - a causa del conflicto desatado- los estudiantes venían de muy lejos y no podían graduarse en la sede del Estudio, sino en la Catedral. Los Paheres solicitaron la intervención del Rey, a fin de que los grados se diesen de nuevo en la Universidad y que calmase los ánimos del cabildo catedralicio ${ }^{109}$, que mantenía una relación difícil con el obispo.

Pocos días después, el 11 de octubre, escribían congratulándose de la buena elección del Magnífico Sr. Cordelles "per a fer bon asiento del Estudi"10".

102 AMP, Reg. 850 (1572-1574), ff. 86v-87r.

103 AMP, Reg. 850 (1572-1574), ff. 87v-90r.

104 Poch, "Del Estudio General...", 120.

105 AMP, Reg. 850 (1572-1574), f. 92r.

106 AMP, Reg. 850 (1572-1574), f. 93r.

107 AMP, Reg. 850 (1572-1574), ff. 93v-94r.

108 AMP, Reg. 850 (1572-1574), ff. 99v-100r.

109 AMP, Reg. 850 (1572-1574), ff. 101v-102v.

110 AMP, Reg. 850 (1572-1574), ff. 103r-104r. 
Miguel de Cordelles, ciudadano honrado y Doctor en derecho, llegó a ser Regente de la Cancillería ${ }^{111}$, y fue enviado a Lérida para inspeccionar personalmente la problemática de la Universidad. Mediante la misiva de los Paheres sabemos que a la sazón el obispo estaba ausente de la Ciudad. Se había refugiado en Monzón, donde -según indica Lladonosa ${ }^{112}$ - acudían los prelados cuando tenían problemas con las autoridades civiles y eclesiásticas.

Para agravar más la situación, Bartomeu Serret, canónigo y catedrático de tercia de cánones, hizo un grave menosprecio al Rector del Estudio. Ello generó un pleito por parte del Rector Joan Baptista Bohil ${ }^{113}$. Vistas las circunstancias, el canónigo y Vicario General Onofre Moliner consideró que Serret merecía por ello ser suspendido de su cátedra ${ }^{114}$.

El Rector y Consejo de la Universidad de Lérida escribieron al Rey el 13 de septiembre de 1574 para que mandase "remediar vn tant gran dany y com ara haiam entes la voluntat de Vostra Magestat y es que dita Universitat torne a son bo y antic stament ${ }^{115}$ ". Asimismo, los Paheres escribieron de nuevo al Virrey Fernando de Toledo indicando que no llegaba el "redreç" de la Universidad y que el Sr. Cordelles ya se había marchado. Se quejaban amargamente de la actitud del obispo: "les malicies grans y odis que lo Sr. Bisbe y los officials an pres tot aquest poble y haver volgut traura la ma a tornar lo Studi al que solie ${ }^{116 "}$.

Los Paheres escribieron el 25 de noviembre de 1574 indicando que el Rector Juan Bautista Bohil había emprendido el viaje hacía dos días, en nombre del Estudio General, hacia la Corte para dar explicaciones directamente al Rey. Pedían al Virrey y al Rey que atendiesen a Bohil y le indicaban los problemas más acuciantes: los lectores tenían que cobrar su salario por Navidad y no sabían si el obispo querría pagar su parte. Los Paheres recalcaban asimismo la necesidad de nombrar administradores para el Estudio ${ }^{117}$.

La situación era muy tensa. Las diferencias entre el Obispo, el Rector y la Ciudad parecían irresolubles, tal y como se desprende de la correspondencia entre el virrey y el vicecanciller Bernardo de Bolea en los primeros meses de $1575^{118}$. Lo cierto es que al final el obispo concluyó los estatutos, que fueron

111 Francisco José Morales Roca, Próceres habilitados en las Cortes del Principado de Cataluña, siglo XVII: 1599-1713, Vol. 1 (Madrid: Hidalguía, 1983), 206.

112 Lladonosa, "Humanisme i Reformes...", 92.

113 ACL, P7_M2_P3_C06, s.f.

114 AMP, Reg. $8 \overline{50}$ (1572-1574), f. 106v.

115 Sánchez-Lauro Pérez, “L’Estudi General de Lleida...”, 268.

116 AMP, Reg. 850 (1572-1574), f. 108r.

117 AMP, Reg. 850 (1572-1574), f. 113r.

118 Esteve Perendreu, "Rentas y reformas...", 50. 
aprobados y sancionados por Felipe II el 27 de julio de dicho año ${ }^{119}$. Nada podemos añadir ya a lo que se ha dicho sobre los estatutos ilerdenses de Antonio Agustín. Han sido comentados con detalle por Esteve Perendreu ${ }^{120}$ y otros y, sin duda, representan una estructuración definitiva de la Universidad, ahondando en las reformas del obispo Despuig.

Como premio a tan ardua labor, Antonio Agustín, que estaba muy bien considerado y mantenía un sostenido epistolario ${ }^{121}$ tanto con Felipe II como con Gregorio XIII, consiguió de ambos su remoción de aquella incómoda sede de Lérida y su promoción al arzobispado de Tarragona. Éste estaba vacante por la muerte del cardenal Cervantes, quien había puesto las bases de la Universidad de Tarragona, a la que Antonio Agustín acabó de dar fisonomía y que fungió como seminario-universidad y como lugar de graduación de muchos clérigos de las diócesis vecinas ${ }^{122}$.

Sentado lo anterior, cabe reflexionar sobre por qué se dio dicho conflicto entre Antonio Agustín y las autoridades ilerdenses. Hay que pensar que, junto con las circunstancias personales, incomprensiones y rencillas, Agustín atrajo a Lérida a muchos humanistas y que se rodeó de ellos durante unos años en los que hubo una gran actividad intelectual en el Estudio. Su concepción de la Universidad estaba muy influenciada por el retorno italiano al cultivo de los clásicos grecolatinos y ambicionaba para la Universidad de Lérida una nómina de profesores eruditos, procedentes de toda la Corona de Aragón.

De la relación de profesores, desconocida hasta ahora, se desprende que Agustín tuvo una concepción intervencionista con respecto a la gestión y a la enseñanza en el Estudio, y que ello dio motivo a quejas por parte de las autoridades civiles y universitarias. Cabe pensar que los Paheres consideraron que el obispo visitador-reformador se extralimitaba en sus atribuciones, si bien la fama intelectual que tenía era, en sí misma, una atracción y un reclamo para los estudiosos, que -bajo la protección episcopal- fueron luego profesores en la Universidad.

119 AMP, Libre del Studi, Reg. 23, ff. 93-98v.

120 Véase Esteve Perendreu, F.: El Régimen jurídico... y también "Rentas y reformas...", 5155 , con abundante bibliografía.

121 Joan Carbonell Manils, "La relación epistolar inédita entre Antonio Agustín y el Papa Gregorio XIII" Faventia 22/2 (2000): 131. Así le agradeció Agustín al Santo Padre su traslado de Lérida: "placuit Philippo Regi nostro Catholico a S(anctita)te V(estra) postulare ut me, qui in hoc minore grege Ilerdensi operam inutilem consumpsi, ceteris praeferat et provinciae totius oves committat".

122 Rafael Ramis Barceló, "Grados mayores en la Universidad de Tarragona (1580-1624)", Analecta Sacra Tarraconensia (en prensa). 
Pese a que no haya quedado constancia manuscrita de su influencia en la promoción académica de Pere Joan Nunyes, Pere Mollan y otros eruditos valencianos mencionados, lo cierto es que el criterio del obispo debió de tener mucho peso en un Estudio que cada vez tenía una proyección más bien local. De hecho, en la Universidad de Lérida desde la reforma de Antonio Agustín hasta su transferencia a Cervera encontramos un profesorado de renombre más bien local o regional, frente a la brillante nómina de lectores que se congregaron cabe el Segre durante el período que hemos tratado aquí.

Sin duda, el enfrentamiento entre Agustín, los canónigos, los Paheres y el Rector fue la chispa que facilitó la marcha de muchos docentes a la Universidad de Barcelona. Con un ambiente hostil y un obispo cuestionado (e incluso exiliado en Monzón), no es de extrañar que algunos de los profesores más reconocidos aceptasen la tentadora oferta de la pujante Universidad barcelonesa, que no sólo les ofrecía buenas condiciones económicas, sino también una cercanía con las instituciones de gobierno que facilitaba su propia promoción. Recordemos que en el curso 1573-1574 enseñaron leyes en la Universidad de Barcelona Jaume Mir, Antoni Oliba y Lluís Fuster y -desde el curso 1575-1576- Joan Castelló, todos ellos procedentes de Lérida ${ }^{123}$. Éstos se añaden a los ya citados (Nunyes, Vilanova...)

En definitiva, no es casual que cuando empezaron los enfrentamientos más serios entre Antonio Agustín, los Paheres y el Estudio General, los profesores se marcharan a otras sedes, particularmente a la de Barcelona, que empezaba a emprender su vuelo y que estaba deseosa de competir con (y vencer a) la aneja Universidad ilerdense.

Asimismo podemos afirmar que la presencia de Antonio Agustín en Lérida significó un momento dorado en cuanto a la nómina de profesores. Sin duda, ninguna otra universidad hispánica -hecha la salvedad de Salamanca, y quizás de Alcalá- gozó de un profesorado tan reputado en aquellos años. Sin embargo, el carácter del obispo, su intervencionismo y sus ideas humanísticas chocaron con la voluntad de muchos estudiantes y de los Paheres, que tenían una visión pragmática que anteponía los equilibrios de poder local (e incluso entre las tres nationes) sobre los ideales humanísticos del obispo.

Al final, Antonio Agustín, tras una dura lucha con las autoridades locales (que tenían unos argumentos económicos y sociales muy convincentes y realistas) concluyó su pontificado reformando la Universidad, consolidando las cátedras existentes y dotándola de una estabilidad institucional que duró -con

123 Fernández Luzón, La Universidad de Barcelona..., 320. 
pequeñas modificaciones- hasta su transferencia a Cervera. El nacimiento de nuevas sedes universitarias (Zaragoza, Tarragona, Vic, Tortosa, Solsona...) y la voluntad oligárquica de la Pahería sobre el control del profesorado impidieron otro período de esplendor docente como el de aquellos años.

\section{CONCLUSIONES}

Hasta ahora se sabía -aunque de forma general e imprecisa- que Antonio Agustín había influido en el Estudio General de Lérida y que se había rodeado de humanistas. Gracias a la documentación manuscrita que aquí hemos analizado conocemos una nómina bastante exacta de los profesores durante su pontificado y, efectivamente, constatamos los vínculos del obispo con muchos de ellos.

Las tres contribuciones más relevantes de este trabajo son la nómina de profesores, la constatación de la presencia de importantes humanistas vinculados con Antonio Agustín, y la aportación de nuevos datos al enfrentamiento que sustuvieron el obispo, los Paheres y las autoridades del Estudio General.

Conocemos mejor ahora la trayectoria de algunos afamados teólogos como Fr. Juan Moreno o Fr. Antoni Pont, y aportamos nuevos datos sobre la ejecutoria de destacados juristas como Antoni Oliba, Pere Mollan, Lluís Fuster, Pere y Francesc Molí, así como también de relevantes filósofos, gramáticos y humanistas, como Miquel Ferrer, Gabriel Massot, Bartomeu Vilanova... De todos ellos tenemos información desconocida acerca de sus lecturas o faltas en ellas.

Apuntamos también la posible conexión valenciana, con el caso especial de Pere Joan Nunyes (de quien confirmamos su estadía en Lérida como lector de artes y de gramática), así como también de Onofre Serra y Vicent Ciurana, de quienes conjeturamos su identificación.

Sin duda, el Estudio de Lérida tuvo durante el pontificado de Antonio Agustín los mejores profesores de la Corona de Aragón, algunos de los cuales acudieron allí expresamente atraídos por la personalidad cultivada del obispo, como se había puesto de manifiesto en alguna documentación. El humanismo intuido y esbozado por Lladonosa puede confirmarse definitivamente con esta nómina, que no deja lugar a dudas.

Y el desencuentro entre el obispo y las autoridades acabó pasando factura al profesorado local o foráneo, que terminó marchándose a la Universidad de Barcelona (Jaume Mir, Antoni Oliba y Lluís Fuster, Joan Castelló, Bartomeu 
Vilanova...) o a otras. Les llevó a Lérida la búsqueda de nuevos horizontes y de libertad, amparada por Antonio Agustín, y la perdieron cuando el obispo empezó a ser cuestionado por las autoridades del Estudio General, los estudiantes y los Paheres. Todos los datos apuntan a que el obispo facilitó la estabilización de algunos renombrados amigos y colaboradores suyos como profesores en el Estudio General, y que el prelado no tenía prisa en la reforma del mismo, sino que prefería atraer a docentes afamados.

Las autoridades querían asegurarse el pago de las rentas que gravaban sobre la mitra, y ansiaban el control de la institución, que desempeñaba un papel importante en la promoción de la oligarquía de la ciudad. Lo que empezó como una desavenencia de intereses y de sensibilidades acabó desencadenando un conflicto que, a la postre, acabó con la promulgación de unos estatutos que blindaban las competencias y las cátedras de la Universidad, y con la remoción del prelado.

Lladonosa interpretó acertadamente la falta de entendimiento de las partes y subrayó la gravedad del conflicto, al involucrar al Rector y a los Paheres, así como también a canónigos, estudiantes, maestros... todos ellos tomaron cartas en un conflicto que concluyó de la mejor forma posible para el Estudio y que fue el final de una etapa, que podría calificarse de tardohumanista, en Lérida.

En este sentido, podemos decir que desde la fundación de la cátedra de teología del obispo Conchillos se vivió una época de esplendor, que se incrementó con las reformas del obispo Despuig y que llegó a su cénit en el pontificado de Antonio Agustín, lapso que -en cuanto a la notoriedad de su profesorado- podemos estimar que fue una época dorada. En los años siguientes, el escolasticismo se sobrepuso al humanismo, el localismo al universalismo y los equilibrios de poder a la auctoritas del obispo.

Por la defensa acérrima que Felipe II hizo del Estudio de Lérida, podemos decir que la institución pudo sobreponerse parcialmente de la crisis de la década de 1570 , y que siguió contando con algunos maestros universitarios notables, de quienes tenemos algunos datos aislados gracias a los trabajos de Lladonosa y especialmente de Esteve Perendreu. El hallazgo de más materiales inéditos, espigando en diferentes fuentes, quizás nos permita conocer el ambiente en el Estudio en los años finales del reinado de Felipe II, en los que se creó la maestrescolía y se consolidaron algunas reformas llevadas a cabo por Antonio Agustín.

En fin, por ahora, este trabajo nos permite conocer con mucho más detalle esta época central del siglo XVI en la Universidad ilerdense, al tiempo que 
ayuda a confeccionar una prosopografía de interés para la historia de la ciudad de Lérida y a establecer muchos vínculos sobre la movilidad del profesorado en las universidades de la Corona de Aragón.

\section{BIBLIOGRAFÍA}

[Aguiló, Mariano], Catálogo de obras en lengua catalana impresas desde 1474 hasta el presente (s.1: 1865).

Alcina Rovira, Juan Francisco. "Los inicios del ramismo enEspaña". En Los humanistas valencianos y sus relaciones con Europa en J. Pérez Dura y J. M. Estellés, (eds.), 117-136. Valencia: Ajuntament de Valencia, 1998.

- "Antonio Agustín y el índice de libros prohibidos del Concilio de Trento (Roma, 1564)". Calamvs renascens: Revista de humanismo y tradición clásica 3 (2002): 7-14.

— "El Humanismo de Antonio Agustín". En Mecenazgo y Humanidades en tiempos de Lastanosa: Homenaje a Domingo Ynduráin en A. Egido y J. E. Laplana (coord.), 31-50. Zaragoza: Institución Fernando el Católico e Instituto de Estudios Altoaragoneses, 2008.

Antonii Augustini archiepiscopi Tarraconensis Opera omnia. Vol. 7. Lucae: Typis Josephi Rochii, 1772.

Bada i Elias, Joan. "Aportacions doctrinals d'Antoni Agustín a la tercera etapa del Concili de Trento (1562-1563)". Revista Catalana de Teologia 12/1 (1987): 125-139.

Balasch, Esther (coord.). Antoni Agustí, bisbe de Lleida i arquebisbe de Tarragona, 1517-1586: aportacions entorn el marc sòcio-cultural de Catalunya en la seva època. Lleida: Publicacions dels Amics de la Seu Vella, 1995.

Barbeito Díez, M. Pilar. Pedro Juan Núñez, humanista valenciano. Tesis doctoral. Madrid: Universidad Complutense, 1996.

Bover, Joaquín M. Biblioteca de autores baleares, vol. 2. Palma: Gelabert, 1868.

Capilla, Andrés. Libro llamado Consuelo de Nuestra Peregrinación de grande utilidad y consolaciòn para todos los fieles, en que se trata de la dignidad y 
exellencia de la Religión Christiana. Lerida: Pedro de Nobles y Juan de Villanueva, 1574.

Carbonell i Manils, Joan. Epigrafia i numismàtica a l'epistolari d'Antonio Agustín (1551-1563), Tesis doctoral, Bellaterra: Universitat Autònoma de Barcelona, 1992.

- "La relación epistolar inédita entre Antonio Agustín y el Papa Gregorio XIII". Faventia 22/2 (2000): 121-138.

Durán Gudiol, Antonio. "Notas para la historia de la Universidad de Huesca en el siglo XVI". Hispania Sacra 21 (1968): 87-150.

Esteve Perendreu, Francesc. El Régimen jurídico del Estudio General de Lleida: s. XIII/XVIII, Lleida: Publicacions de l'Estudi General de Lleida-Pagès, 1992.

- "La docència de la teologia a Lleida, la càtedra del bisbe Conchillos i les altres càtedres teològiques de l'Estudi General". En El bisbe Jaume Conchillos, l'humanisme a Catalunya, X. Company (ed.), 141-179. Lleida: Amics de la Seu Vella, 1993.

— "Rentas y reformas del Estudio General de Lérida", Analecta Sacra Tarraconensia 69 (1996): 29-86.

- Mestrescoles i rectors de l'Estudi General de Lleida (1597-1717). Lleida: Edicions de la Universitat de Lleida, 2007.

- El Col-legi universitari de l'Assumpció de Santa Maria de Lleida (segles $X I V$-XIX). Lleida: Universitat de Lleida, 2010.

Falcón Pérez, Maria Isabel, Motis Dolader, Miguel Ángel. Procesos criminales en el Arzobispado de Zaragoza. Zaragoza: Gobierno de Aragón, 2000.

Felipo Orts, Amparo. La Universidad de Valencia durante el siglo XVI (14991611). Valencia: Universidad de Valencia, 1993.

Felipo Orts, Amparo; Peris Felipo, Francisco J.; Miralles Vives, Francisca. Estudiantes y probanzas de cursos en la Universidad de València (15611707). Valencia: Universidad de Valencia, 2013.

Fernández Luzón, Antonio. La Universidad de Barcelona en el siglo XVI. Barcelona: Universitat de Barcelona, 2005.

Finestres y de Monsalvo, Jaime. Historia del Real Monasterio de Poblet, vol. 4. Tarragona: Joseph Barber, 1756. 
Fuster, Justo Pastor. Biblioteca Valenciana de los escritores que florecieron hasta nuestros dias, vol. 1. Valencia: Ximeno, 1827.

Gallego Salvadores, Jordán. "La metafísica en España durante el siglo XVI". En Repertorio de las ciencias eclesiásticas en España, VII, 142-234. Salamanca: UPSA, 1979.

Gayá Massot, Ramon. "Provisión de cátedras en el Estudio General de Lérida", Analecta Sacra Tarraconensia, 30 (1958): 233-296.

Grau, Ferran. Las Retóricas de Pedro Juan Núñez. Tesis doctoral (Valencia: Universidad de Valencia, 1994).

Gutiérrez, Constancio. Trento, un problema: la última convocación del concilio (1552-1562), V, Madrid: Universidad Pontificia de Comillas, 2000.

Latassa, Felix de. Biblioteca Nueva de los escritores aragoneses: que florecieron desde el año de 1500 hasta 1599, Vol. 1 (Pamplona: en la oficina de Joaquín Domingo, 1798).

Lladonosa, Josep. La Facultat de Medicina de l'antiga Universitat de Lleida. Lleida: Rafael Dalmau, 1969.

- L'Estudi General de Lleida del 1430 al 1524. Barcelona, Institut d'Estudis Catalans, 1970.

- "Humanisme i Reformes a l'Estudi General de Lleida durant el segle XVI" en AAVV, VIII Congreso de Historia de la Corona de Aragón, III, 2, 87106. Valencia: Universidad de Valencia, 1973.

— Història de Lleida. vol. II. Tàrrega, F. Camps Calmet Editor, 1974.

— Lérida medieval. Tomo II (siglos XIV-XV). Lérida: Dilagro, 1975.

— Lérida moderna. Epoca de los Austrias. Lérida: Dilagro, 1977.

- Lérida moderna. Epoca de los Borbones. Lleida: Dilagro, 1980.

— Història de Lleida. II, Lleida: Dilagro, 1991.

Madurell Marimon, Josep M. "Luis Juan Vileta”. Analecta Sacra Tarraconensia 37 (1964): 19-76.

Martínez Rodríguez, Miguel Ángel. "Linaje y poder en la Cataluña foral: la actividad política de los Copons”. Cuadernos de Historia Moderna 22 (1999): $11-31$. 
Massot, José. Compendio Historial de los Hermitaños de nuestro Padre San Agustín del Principado de Cataluña. Barcelona: Juan Jolis, 1699.

Molini, Francisci. De brachio seculari Ecclesiae praestando et mutuis iudicum auxiliis celeberrimi commentarij: in tres libros distincti; his accessit liber singularis De sacra homicidio amittenda immunitate nec ne. Barcelona: Ioannem Simon, 1607.

- Tractatus celebris, et insignis de ritu nuptiarum, et pactis in matrimonio conuentis. Barcelona: Laurentij Déu, 1617.

Morales Roca, Francisco José. Próceres habilitados en las Cortes del Principado de Cataluña, siglo XVII: 1599-1713, Vol. 1. Madrid: Hidalguía, 1983.

- Ciudadanos y burgueses honrados habilitados como Sindicos del Brazo Real en las Cortes del Principado de Cataluña: dinastías de Trastámara y de Austria, siglos XV y XVI, 1410-1599. Madrid: Hidalguía, 1995.

Narváez Cases, Carme. "El patronatge de les noves oligarquies urbanes a l'art català dels segles XVI i XVII", Recerques 51 (2005): 5-25.

Pemán Gavín, Juan. "Los estatutos fundacionales de la antigua universidad Ilerdense (año 1300)", en J. J. Busqueta (ed.), Llibre de les Constitucions $i$ Estatuts de l'Estudi General de Lleida, 25-53. Lleida: Universitat de Lleida, 2000.

Peset, Mariano. "La fundación y el fuero universitario de Lérida". Hispania, 199 (1998): 515-536.

Planas Rosselló, Antonio. La Real Audiencia de Mallorca en la época de los Austrias (1571-1715). Barcelona: Publicacions de la Universitat Pompeu Fabra, 2010.

Poch, José. "Del Estudio General de la Universidad de Lérida. Carta de los Paheres a Felipe 11 (17 agosto 1574)". Ciudad, 26 (1974): 120.

— "El rectorado del Estudio General de Lérida en la anualidad 1573-1574". Ciudad, 27 (1975): s.n.

Ramis Barceló, Rafael. "Los grados en teología en la Universidad de Barcelona durante el siglo XVI". Anuario de Historia de la Iglesia 24 (2015): 291-309.

— "Los graduados en Leyes y Cánones de la Universidad de Barcelona durante el siglo XVI". Anuario de Historia del Derecho Español 85 (2015): 475496. 
- Doctores hispanos en Leyes y Cánones por la Universidad de la Sapienza de Roma (1549-1774). Madrid: Dykinson, 2017.

— "Grados mayores en la Universidad de Tarragona (1580-1624)". Analecta Sacra Tarraconensia. (en prensa).

Ruiz, Elisa: "Los años romanos de Pedro Chacón". Cuadernos de Filología Clásica 10 (1976): 189-247.

Sainz de Baranda, Pedro. España Sagrada, T. XLVII. Madrid: Imprenta de la Real Academia de la Historia, 1850.

Sanahuja, Pedro. "La Universidad de Lérida y los franciscanos". Archivo Iberoamericano 7 (1947): 167-242.

Sánchez-Lauro Pérez, Sixto. “L'Estudi General de Lleida davant un mo-ment històric d'inflexió renovadora: peticions locals d'intervenció regi a Felip II". Revista de Dret Històric Català 9 (2009): 253-269.

Serra i Puig, Eva. Els Llibres de l'Ànima de la Diputació del General de Catalunya (1493-1714). II. Barcelona: IEC, 2015.

Vázquez Janeiro, Isaac. "Repertorio de franciscanos españoles graduados en teología durante la Edad Media", en Repertorio de Historia de las Ciencias Eclesiásticas de España, vol. 3: Siglos XIII- XVI, 235-320. Salamanca: UPSA, 1971.

Villanueva, Jaime. Viage literario a las iglesias de España, T. XVII. Madrid: Imprenta de la Real Academia de la Historia, 1851.

Viola i González, Ramiro. "Un testament canonical (16 d'agost de 1561)", Analecta Sacra Tarraconensia 71 (1998): 907-920. 